\title{
WILLIAM MARÇAIS Y SU OBRA SOBRE EL DIALECTO ÁRABE HABLADO EN TREMECÉN. TRANSCENDENCIA PARA LA HISTORIA DE LA DIALECTOLOGÍA DE ARGELIA
}

\author{
SARRA IKRAM HADEF \\ Universidad Abou Bekr Belkaid de Tremecén
}

\begin{abstract}
Resumen
Breve presentación de la figura del arabista francés William Marçais y sus estudios sobre el dialecto árabe de Tremecén, estudiando el contenido de la obra Le dialecte arabe parlé à Tlemcen. Grammaire, textes et glossaire (1902) y su labor pionera en la constitución de una dialectología moderna argelina.
\end{abstract}

Palabras clave: William Marçais, dialectología, Tremecén, árabe argelino.

\begin{abstract}
Short introduction to the life of the French Orientalist William Marçais and his works about the Arabic dialect of the region of Tlemcen, focusing attention on the contents of Le dialecte arabe parlé à Tlemcen. Grammaire, textes et glossaire (1902). We remark his pioneering career in the consolidation of the modern Algerian Dialectology.
\end{abstract}

Keywords: William Marçais, Dialectology, Tlemcen, Algerian Arabic.

\section{APROXIMACIÓN A LA DIALECTOLOGÍA ARGELINA}

Actualmente los dialectos pueden ser herramientas para permitir una transferencia efectiva del patrimonio cultural, más allá de las limitaciones impuestas por las lenguas. Lingüísticamente, el dialecto se define como proceso diatópico, porque exige una adecuada caracterización del idioma al territorio. Además, el concepto "dialecto" hace referencia a una de las posibles variedades de una lengua. En este contexto en el cual se desarrolla la dialectología como disciplina científica hay que situar el estudio histórico y lingüístico del árabe tremecení y, en general, de la la dārîya (dialecto argelino y magrebí), que ha sido socialmente un tabú durante mucho tiempo. 
El dialecto o el habla pueden ser variedades sociales o contextuales tanto como geográficas. La descripción lingüística externa pretende representar, de forma estructural, el conocimiento necesariamente en sus diferentes dimensiones, es decir, temporal, geográfica, social y pragmática. La dialectología por su parte utiliza contextos especializados de las disciplinas lingüísticas en general, y la fonética en especial, de una lengua, en una región, durante un tiempo determinado o durante varios periodos de forma comparada ${ }^{1}$.

En época moderna el estudio de las variedades de la lengua árabe ha conocido un desarrollo notable. Los dialectos han convivido sin ningún estatus reconocido al lado de una de las lenguas mundiales más prestigiosas e institucionalizadas. La clasificación diatópica o geográfica de los dialectos árabes en la época moderna distingue los hablados en la región oriental de los de la región occidental. Los dialectos occidentales se extienden en regiones del este de Egipto, Libia, Argelia y Marruecos, lugares estos últimos donde existe una mezcla del habla beréber, árabe y andalusí. Este fenómeno es sin duda diglósico. La diglosia se comprende como una situación de convivencia de dos variedades lingüísticas en una misma población o territorio; es decir que la diglosia designa a la convivencia de dos o más lenguas distintas con un rango de uso diferente en una misma zona geográfica ${ }^{2}$.

En cuanto a la actual situación dialectológica de Argelia, existen variedades en cada región. Generalmente los dialectos argelinos se caracterizan por una silaba cerrada en la primera vocal, sobre todo en regiones beduinas como Nedroma en Tremecén ${ }^{3}$. En las regiones del noreste la primera radical, por ejemplo con consonante liquida, también afecta a la cantidad de la vocal. En fin, en este breve trabajo queremos simplemente esbozar la problemática en torno a la situación de los dialectos de la lengua árabe, y destacar la figura de William Marçais como pionero en el estudio de la dialectología argelina.

${ }^{1}$ Véase Francisco Gimeno Menéndez, Dialectología y sociolingüistica españolas, Alicante, Universidad de Alicante, 1990.

${ }^{2}$ Cf. Moisés Garduño, "Corán y lengua árabe, entre el dialecto, el árabe medio y el fuṣhă", Estudios de Asia y África, 2012, vol. XLVII, num.1, pp.164-165.

${ }^{3}$ Cf. F. Javier Brage González, Estudios sobre el vocalismo en los dialectos árabes, Madrid, Instituto Hispano-Árabe de Cultura, 1988, p. 44. 


\section{SEMBLANZA DE WILLIAM MARÇAIS}

William Marçais fue un arabista orientalista francés, el primer investigador que ha realizado investigaciones sobre los dialectos árabes en el norte de África, desde Tánger en Marruecos, a Tremecén en Argelia. En 1935 el director de francés de origen beréber M. Ben Embarek contaba que cuando era estudiante en París, fue en varias ocasiones a escuchar a William Marçais al Colegio de Francia, donde se consagraban sus enseñanzas sobre el habla árabe de Argelia ${ }^{4}$.

$\mathrm{Su}$ nombre completo era William Ambroise Marçais, nació en 1872 en Rennes, y murió en 1956 en París. Fue lingüista y arabista, especialista en dialectología magrebí, fue doctor en Derecho en 1898, fue director de la madrasa de Tremecén (1898-1904), inspector general de educación primaria indígena en Argel (1909-1913), graduado en la catedral arábiga del Magreb de la Escuela de lenguas orientales (1920-1927). Profundizó su conocimiento del árabe dedicando su tiempo al estudio de los antiguos escritores de la Edad Media. Además fue profesor en el Colegio de Francia (1927-1942), fue miembro del instituto académico de «Les inscriptions des Belles Lettres», elegido en 1927. Al mismo tiempo estudió los modismos populares, publicando sucesivamente importantes estudios sobre los dialectos de Tánger, Tremecén, Saida y Gabes.

W. Marçais estudió el lenguaje y el vocabulario del pequeño pueblo de Tarkouna en Túnez, lo cual lo hizo ser famoso. Estos estudios tenían como fin mostrar la extensión de la dialectología, haciendo un glosario que le llevó completar un año; cada palabra de este trabajo fue estudiada desde su etimología hasta sus diversos significados.

Actualmente su figura está siendo reivindicada por parte de algunos escritores, como Mélanges offerts à William Marçais par l'Institut d'études islamiques de l'Université de Paris ${ }^{5}$, y también desde Argelia, por ejemplo por Fatima Zohra Bouzina en su obra titulada Tlemcen Capital musulmane, le siècle d'or du Maghreb Central (Argel, 2011).

\footnotetext{
${ }^{4}$ Robert Laffitte, "In memoriam: William Marçais", l'Algérianiste, 1986, núm. 36: <http://www.cerclealgerianiste.fr $>$.

${ }^{5}$ G. P. Maisonneuve, Mélanges offerts à William Marçais par l'Institut d'études islamiques de l'Université de Paris, París, Université de Paris, 2009.
} 
III. LA OBRA LE DLALECTE ARABE PARLE À TLEMCEN. GRAMMAIRE, TEXTES ET GLOSSAIRE

En cuanto a los estudios de los dialectos árabes argelinos, Williams Marçais realizó una obra capital: Le dialecte arabe parlé à Tlemcen. Grammaire, textes et glossaire. El autor hace estudios sobre el uso del habla árabe en la zona de Tremecén, ciudad que se encuentra en el noroeste de Argelia, y ciertamente es un lugar privilegiado por su civilización e historia.

La obra de W. Marçais presenta en primer lugar un bosquejo gramatical del dialecto de Tremecén. En segundo lugar muestra una serie de textos de prosa, poesía y folclore. El último apartado recoge un glosario en el cual se explican los términos que están anotados con asterisco en los textos árabes de cuentos y canciones infantiles. En fin, la obra es resultado de sus estudios araboislámicos en Tremecén durante cuatro años, incluyendo documentos, léxico, gráficos, cuadros gramaticales y sociolingüísticos.

Me gustaría presentar, a título de ejemplo, el sistema fonético que emplea y los signos en la transcripción para el estudio del dialecto de Tremecén, para que el lector pueda hacerse una idea de los recursos lingüísticos presentados en la obra.

1.Transcripción del dialecto de Tremecén en los estudios de W. Marçais

En la obra Le dialecte arabe parlé à Tlemcen Grammaire; textes et glossaire, Williams Marçais emplea unos determinados signos de transcripción para reflejar sus muestras de lengua y llevar a cabo sus estudios históricos y sociolingüísticos del dialecto de Tremecén, de la manera siguiente ${ }^{6}$ :

-Para las consonantes:

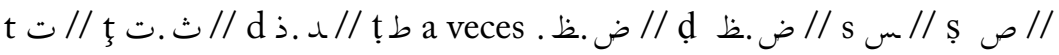

$$
\begin{aligned}
& \text { s }
\end{aligned}
$$

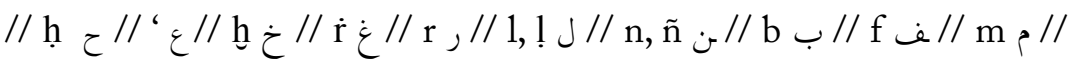

\footnotetext{
${ }^{6}$ París, Ernest Leroux, 1902, p. 9.
} 
-Para las vocales:

a (a pura) // ā (ai en francés) // à (inclinándose hacia o) // ạ (inclinándose hacia el diptongo an, in en lengua francesa) // o ( o pura) // o (inclinándose hacia u) // ô (entre o y oe en francés) // u (ou francés) // e (e pura) // è-ẹ (entre i-e) // i (i pura) // $\bar{a}-\bar{e}-\bar{i}-\bar{o}-\bar{u}$ (largas y no acentuadas) // à- $\dot{o}$ etc. (largas y acentuadas) // â-ô etc. (breves y acentuadas) // ă-ǒ-ě (muy breves).

En este sistema fonético, W. Marçais incluye el signo — para indicar la formación del diptongo, como en au-ou ${ }^{7}$.

En cuanto al vocalismo como puede verse, se sigue el siguiente modelo: v: vocal breve no acentuada // $\breve{v}$ : vocal muy breve // $\bar{v}$ : vocal larga no acentuada

De manera general, entre los dialectos del oranesado y el estudio del árabe tremecení, aparece como hablante claramente identificado el ciudadano urbano. En el Magreb central la distinción de dialectos se divide en dos grandes clases: beduinos o rurales y ciudadanos o civilizados.

Para aclara más, William afirma que los dialectos ciudadanos tienen el sonido /Qa/ para a mientras que los dialectos beduinos tienen / $\mathrm{Ga} /$ para . De otro modo, los dialectos ciudadanos ofrecen una pronunciación /U/ del pronombre masculino singular, mientras que los dialectos beduinos ofrecen una pronunciación /Ah/ /Oh/. También, los ciudadanos reducen a Mafācil, Facālil sin ì larga. Los dialectos urbanos ofrecen el plural de los verbos defectuosos con de las terminaciones $\bar{i} u, \bar{a} u, y$ otros ofrecen simplemente $u$, û. Los beduinos de la región de Tremecén suelen usar las vocales largas en última consonante de palabra, por ejemplo: /barkāy/ ¡vale!; /drārī/ los niños; /ṣrīr/ pequeño; /bdāwu/ empiezan (ellos, ellas).

Marçais señala que el dialecto tremecení es el único dialecto oranesado que ofrece las características de los dialectos urbanos. Al fin el autor acaba su estudio mostrando textos en prosas, poesías infantiles y un glosario general.

${ }^{7}$ Ibid., p. 128 


\section{Normas gramaticales}

En la primera parte de su obra, W. Marçais establece las normas gramaticales del dialecto árabe hablado en Tremecén. En primer lugar muestra la segunda persona femenina de los verbos regulares, que tienen la terminación $\overline{1}$, en el modo indicativo e imperativo:

- En la $2^{\text {da }}$ persona femenina: takatbī (presente), ktabtī (pasado), katbī (imperativo).

En este punto, el arabista presenta la clasificación de verbos sordos médd (dar), los verbos de hamza (ämén), verbos asimilados uṣöl (llegar), verbos defectuosos qlă (freír), nsă (olvidar), y otras formas de verbos irregulares del dialecto, comparándole con sus estudios del habla de Túnez.

Por otro lado W. Marçais señala que las formas sustantivas de regiones de Túnez se encuentran en el dialecto de Tremecén, indicando que hay una relación natural: "Ici le tlemcenien, comme sur plusieurs autre points, passe par-dessus le tunisien pour donner la main au tripolitaine" ${ }^{8}$. En este capítulo los estudios morfológicos se basan sobre los nombres personales del dialecto tremecení, analizando su forma y su anexión. Concluye señalando que la anexión de sufijos vocálicos determinados por vocales tiene el mismo fenómeno determinado por la conjugación por la anexión de terminaciones vocálicas. La anexión de sufijos consonánticos mantiene el acento en la última silaba.

También, presenta en su obra el número dual que es una de las posibles formas del número gramatical, y expresa la cantidad dos. Contrasta casi siempre con el singular y plural. Así, yẹddîya (mis manos) - heddîk (tus mejillas) - rejlîh (su pie) - wudnîna (nuestras orejas) - jenḥehum (sus alas) ${ }^{9}$.

Sigue el estudio de nombres cardinales, nombres ordinarios, preposiciones, pronombres interrogativos, relativos y demostrativos, pronombres indefinidos y adverbios. Explica con ejemplos recogidos en colaboración con sus alumnos, en conversaciones cotidianas, y en un trabajo de campo realizado en su larga presencia en la ciudad de Tre-

\footnotetext{
${ }^{8}$ Ibid., p. 127.

${ }^{9}$ Ibid., p. 145.
} 
mecén. A título de ejemplo reproducimos los numerales, preposiciones e interrogativos:

- Números cardinales:

wâḥad (uno) - zéuj (dos) - tlâţa (tres) - réb 'a (cuatro) -hémsa (cinco) - sétţa (seis) -séb a (siete) - ţménya (ocho) - ţés 'öd (nueve) - 'áśra (diez) - hŏọảś (once) - ţnàś (doce) -ţ̣öţţàś (trece) - rba ţàś (catorce) hmöṣţàś (quince) - șöţ̧̧aś (dieciséis) - sba ţ̧àś (diecisiete) -ţmenţáś (dieciocho) - 'öśin (veinte) - tläţin (treinta) - reb ện (cuarenta)hemsin (cincuenta) - setţin (sesenta) - seb 'ện (setenta) - ţmạnyin (ochenta) - tes 'ện (noventa) -mǐyá (cien) - âlef (mil) ${ }^{10}$.

- Números ordinales:

éuwöl (primero) - ţảni (segundo) - ţàleţ (tercero) - rảba' (cuarto) hảmes (quinto) - sảţeţ (sexto) - sảba' (séptimo) - ţảmen (octavo) ţàsa' (noveno) - ‘àśer (décimo) ${ }^{11}$.

- Preposiciones simples:

Bï (por, con) - lï (para, a) - fi (en) - kï, kïf (como, tanto...como) dyâl (de) - qûbâla (enfrente) - qọddâm (a lado) - dâhel (interior) hârej (exterior) ${ }^{12}$.

- Pronombres interrogativos:

Śkûn (¿Quién?) - límen (¿a quién?) - ’ălǐmen (¿de quién?) - âsem (¿qué? $)^{13}$.

\section{Patrimonio literario}

W. Marçais presenta muestras de haufī, transcrito como le háúfi, que es una pieza de poesía. Tiene el sentido de $a l-r \bar{u} b \bar{\imath}$, es decir, un tipo de poesía vulgar en la sociedad de Tremecén que se canta en bodas y lugares públicos. Entre las zonas argelinas, la ciudad de Tremecén es conocida como lugar clásico del háúfi, es su pieza más original y representativa. Históricamente el nombre del háúfi no era conocido

\footnotetext{
${ }^{10}$ Ibid., p. 155.

${ }^{11}$ Ibid., p. 161.

12 Ibid., p. 163.

${ }^{13}$ Ibid., p. 173.
} 
en el pasado, por ejemplo Ibn Jaldūn lo cita simplemente como mawāl. ${ }^{14}$

Entre las poesías de háufi que han sido recogidas por W. Marçais, podemos reproducir por ejemplo la siguiente:

Tlemsâne yā' âlìya mémmelhak lessuknân

Fik bnâu èlhădàr wubnâțe qôrörlăn

Fik elbnâțelmelâh $\quad$ ẹgdiu kèlbellâr

Ěmkahhălîn èl'öyûn ěmharqeșên leŝfâr.

Tremecén, la gran ciudad ¡Como es deliciosa tu estancia!

Posees mujeres cultas, mujeres como princesas.

Posees mujeres hermosas, brillantes como el cristal.

En los ojos negro kohol, y cejas unidas por la línea de la aljaba ${ }^{15}$.

Además reproduce textos narrativos, sacados por informantes urbanos, quienes solían contar temas históricos y culturales en dialecto tremecení, tales Elmsîd (escuela coránica), Bâb keššut (La puerta de kaššūt), o Kifâš déblu țúrk 'alā Tlemsân (Cómo los turcos ocuparon Tremecén).

La sección acaba con canciones infantiles y un glosario al final de la obra, explicando el uso del vocabulario árabe hablado en Tremecén. Una de las canciones más famosas en la sociedad de Tremecén es la nana infantil cantada en el mes de ramadán en grupo, cuando los niños ven que alguien no ayuna:

Yã wukkâl ramdân

Yā hậ̂̆ör dinu

Ukélb èssoudân...

Quien no haga el ramadán

que abandone su religión

como el perro de Sudán ${ }^{16}$.

Más allá de los estudios filológicos de W. Marçais que tratan de conocer la gente a través de su lenguaje, su contacto con los árabes

\footnotetext{
${ }^{14}$ Ibid., p. 209.

${ }^{15}$ Ibid., p. 213. Traducción nuestra.

${ }^{16} \mathrm{Ibid}$.
} 
fue siempre productivo. Sin duda, no solamente fue un hombre que sabía el idioma, sino también la psicología de la gente del Magreb central en su diversidad. La obra de W. Marçais nos permite, en nuestros días, acercarnos a los principales rasgos fonéticos y morfológicos del dialecto árabe hablado en Tremecén, su producción literaria y su léxico. A partir de este trabajo pionero, la moderna disciplina dialectológica puede sin duda profundizar más en un tema a todas luces necesario para conocer la verdadera realidad cultural del mundo argelino. 


\section{APÉNDICE ICONOGRÁFICO:}

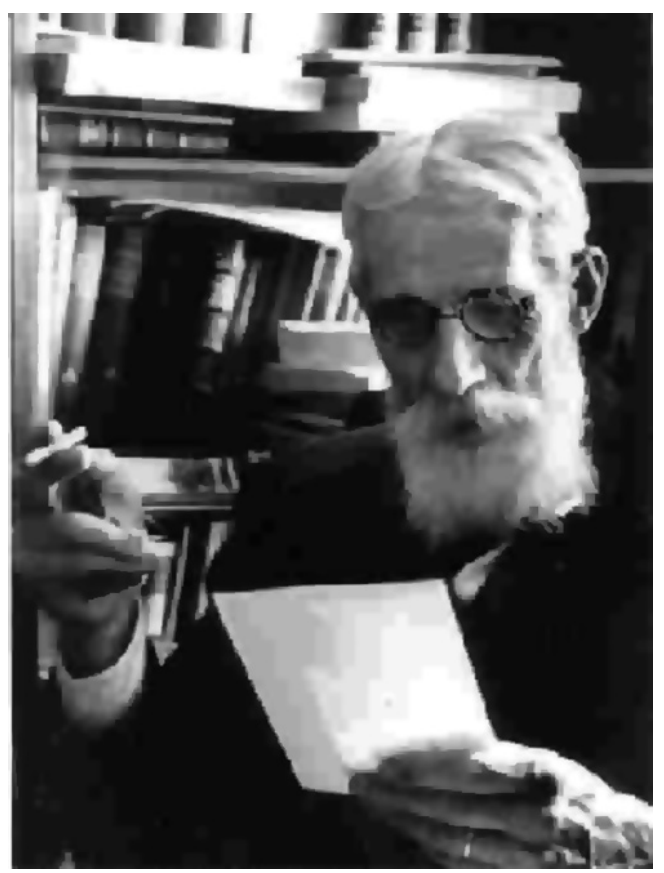

Imagen en Merlin Alfred, "Notice sur la vie et les travaux de M. William Marçais, membre de l'Académie”, Comptes rendus des séances de l'Académie des Inscriptions et Belles-Lettres, 1957, $101^{\text {e }}$ année, núm. 4, p. 402. 




